COGNitiVe STUdies | Études COGNitives, 11

SOW Publishing House, Warsaw 2011

\author{
MAŁGORZATA NOWAKOWSKA ${ }^{1}$, DENIS APOTHÉLOZ ${ }^{2}$ \\ ${ }^{1}$ Université Pédagogique de Cracovie. Pologne \\ (M.Nowakowska@up.krakow.pl) \\ ${ }^{2}$ Université de Nancy 2. France \\ (Denis.Apotheloz@univ-nancy2.fr)
}

\title{
NOTE SUR L'ADVERBE TEMPOREL JUŻ ET SES CORRESPONDANTS FRANÇAIS
}

\begin{abstract}
Generally speaking, this contrastive study of the Polish temporal adverb $j u \dot{z}$ and the French déjà is based around the idea that już and déjà have an invariant meaning but it is also true that they follow different idiosyncratic usages. This article has two parts. First, the authors show the usages of $j u \dot{z}$ which are equivalent to those of déjà. These usages appear when już and déjà are put in a specific verbal context. The authors describe the four cases in which już and déjà are equivalent: "precocity of event", existential usage, iterative-continuative usage, experiential perfect usage. Secondly, the authors analyze four cases in which the Polish adverb juz can't be translated by the French déjà. These incompatibilities can be explained by either morphological idiosyncrasy or the conventionalization of deictic meaning of juz, which is absent of déjà.

Keywords: contrastive study, Polish and French, aspectual-temporal Adverbs, już and déjà, Perfect, temporal deixis.
\end{abstract}

\section{Introduction}

Une analyse générale de tous les emplois de l'adverbe już, dont le fonctionnement est particulièrement complexe, dépasse largement le cadre de cet article. Nos réflexions sur le contraste entre już polonais et déjà français se limiteront par conséquent aux principaux emplois incluant la dimension temporelle. L'un des objectifs de cet article est de montrer que, à côté de quelques emplois où już et déjà peuvent être considérés comme des équivalents, już a conventionnalisé des valeurs spécifiques que déjà ne permet pas de traduire.

\subsection{L'adverbe $j u \dot{z}$ dans les études polonistes}

Curieusement, les monographies de Grzegorczykowa (1975) et de Grochowski (1986) consacrées aux adverbes et particules polonais ne nous apprennent pratiquement rien sur l'adverbe już. Seul Bańkowski $(1971,1976,1977)$ en donne une 
analyse relativement complète. Il écrit que juz entre dans deux types d'oppositions :

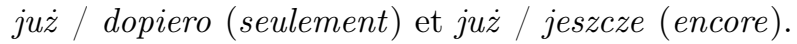

Contrairement à dopiero (seulement), qui signifie le retardement, l'adverbe juz signifie l'accélération. Citons les exemples de Bańkowski (1976), que nous avons traduits en français :

(1) Wrócę już o piątej.

(1a) Je rentrerai déjà à 5 h. ${ }^{1}$

(2) Wrócę dopiero o piątej.

(2a) Je rentrerai seulement à $5 \mathrm{~h}$.

Contrairement à jeszcze (encore), qui signifie la continuation de la situation, już signifie la clôture de l'intervalle couvert par la situation ${ }^{2}$ (wskaźnik prekluzji). Bańkowski illustre cette analyse par les exemples d'énoncés affirmatifs positifs et négatifs :

(3) Janek jeszcze śpi.

(3a) Janek dort encore.

(4) Janek jeszcze nie śpi.

(4a) Janek ne dort pas encore.

(5) Janek już śpi.

(5a) Janek dort déjà.

(6) Janek już nie śpi.

(6a) Janek ne dort plus.

Quant à nous, nous ne pensons pas que już ait une autre interprétation dans (1) que dans (5) et (6). Confronté à dopiero (seulement) ou jeszcze (encore), już signifie toujours que la situation dénotée par le verbe a lieu plus tôt que prévu, emploi que nous appelons « survenance précoce », à l'instar du Trésor de la Langue Française informatisé (voir infra).

Wierzbicka (1969:50) ${ }^{3}$, dans le cadre de sa théorie des primitifs sémantiques, donne une analyse un peu différente. Selon cet auteur, juz a les deux représentations sémantiques suivantes :

$1^{o}$ 'nie sądź, że później' ('ne crois pas que (ça soit) plus tard')

\footnotetext{
${ }^{1}$ Sans mention particulière, les exemples ont été traduits par les auteurs.

2 Nous employons le terme de situation pour désigner tout type de procès, aussi bien statifs que non statifs, téliques que non téliques, instantanés que duratifs, etc.

${ }^{3}$ Cf. la description de już donnée par Pasicki (1976), qui traite de sa correspondance avec l'anglais.
} 
$2^{o}$ 'sądzę, że wiesz, że można było sądzić, że będzie później' ('je crois que tu sais qu'on pourrait croire que ça soit plus tard')

Wierzbicka illustre ces deux représentations sémantiques respectives par les exemples suivants :

(7) Przyszedł już o piątej.

(7a) Il est arrivé déjà à 5 h.

(8) Już przyszedł.

(8a) Il est déjà arrivé.

Comme on le remarque, Wierzbicka essaie de distinguer deux interprétations de $j u \dot{z}$. Cela dit, nous pensons qu'il s'agit bel et bien d'un même $j u \dot{z}$, celui que nous appelons « survenance précoce ». Par ailleurs, son second exemple a au moins deux interprétations : il peut illustrer soit la survenance précoce de la situation soit le caractère effectif de l'accomplissement de la situation.

\subsection{L'adverbe déjà dans les travaux romanistes}

La littérature sur l'adverbe déjà est beaucoup plus abondante que celle sur l'adverbe już (Muller 1975; Hoepelman \& Rohrer 1980; Martin 1980; Franckel 1989 ; Fuchs 1988; Paillard 1992; Nøjgaard 1992-95, Mosegaard Hansen 2000, 2002a, 2002b, 2008; Tahara 2006, Buchi 2007; Apothéloz \& Nowakowska à par. a, Apothéloz \& Nowakowska à par. b). Sans entrer dans le détail des différentes approches, on peut observer que les linguistes distinguent principalement deux valeurs aspectuo-temporelles de déjà : l'une que nous appelons « survenance précoce » et l'autre fréquemment appelée 《itérative » et que nous préférons, quant à nous, qualifier de « déjà de parfait d'expérience », pour éviter toute confusion avec un autre emploi sur lequel nous reviendrons plus $\operatorname{loin}^{4}$. Les exemples suivants que donne Franckel (1989) illustrent respectivement ces deux valeurs :

(9) Tu as déjà mangé tes blinis?! (Franckel)

(10) Tu as déjà mangé des blinis? (Franckel)

Ces deux emplois peuvent être caractérisés sommairement comme suit. La première valeur indique que la situation dénotée par la prédication a lieu plus tôt que prévu; sa négation est ne ... pas encore et elle se combine difficilement avec le passé simple. La seconde valeur indique que la situation a eu lieu une fois au moins, sa négation est ne ... (encore) jamais, elle se manifeste exclusivement en association avec les temps composés.

Quant à déjà de survenance précoce, Muller (1975 : 30-31) lui ajoute encore une caractéristique : la situation dénotée par la prédication est orientée. Ainsi, les heures s'additionnent les unes aux autres ou bien la vie précède la mort et non l'inverse.

\footnotetext{
${ }^{4}$ Cette appellation est justifiée par le fait que cet emploi de déjà est systématiquement associé à un emploi du passé composé habituellement appelé « parfait d'expérience » et traduit en polonais par le passé imperfectif.
} 
(11) Il est déjà 4 heures (*encore) (Muller)

(12) Il est déjà tard (*encore) (Muller)

(13) Il est encore tôt (*déjà ) (Muller)

(14) Il est déjà mort (*encore) (Martin)

Par ailleurs, ces exemples montrent qu'il y a un point commun dans la méthode d'analyse de już polonais et de déjà français : leur description est toujours faite en rapport avec d'autres adverbes aspectuo-temporels comme encore (jeszcze), toujours (zawsze, jeszcze), enfin (wreszcie), etc.

Bien que plus nombreux que les travaux sur już polonais, les travaux sur déjà n'échappent pas à une certaine confusion aussi bien dans les typologies et les appellations proposées que dans l'interprétation des exemples. Même si nous nous inspirons de certaines analyses ou appellations trouvées dans la littérature, nous proposons une autre perspective pour analyser ces adverbes. Nous préférons le terme d'emploi à celui de valeur, en étant convaincus que l'approche monosémique convient mieux ici que l'approche polysémique (cf. Apothéloz \& Nowakowska à par. a, Apothéloz \& Nowakowska à par. b). Suivant cette approche, les adverbes $j u \dot{z}$ et déjà reçoivent une interprétation qui découle de la combinaison de leurs propriétés sémantiques avec des indices pragmatiques et aspectuo-temporels, notamment la forme verbale. Cette dernière, à son tour, s'interprète non de façon indépendante mais toujours en relation avec ces adverbes. Notre analyse s'appuie donc sur la combinatoire de marques aspectuo-temporelles, qui, par elles-mêmes, sont sous-déterminées.

Dans cet article nous avançons une hypothèse de travail concernant l'étude contrastive, hypothèse selon laquelle l'adverbe już a le même sens invariant que déjà. La conséquence en est que już partage certains emplois de déjà et vice versa. Nous présenterons d'abord les cas dans lesquels już se laisse traduire par déjà et ensuite les cas dans lesquels ce n'est pas possible.

\section{Correspondance entre już et déjà}

Dans la présente section nous allons traiter d'emplois dans lesquels déjà correspond exactement à $j u \dot{z}$.

\subsection{Już de survenance précoce}

Comme nous l'avons dit plus haut, cet emploi peut être glosé par « plus tôt que prévu » (Franckel $1989: 265$ à propos de déjà). L'adverbe signale le décalage entre l'écoulement réel du temps et sa perception, ce que nous observons dans les exemples cités ci-dessous :

(15) Już dziesiąta! Muszę się pospieszyć.

(15a) Déjà 10h! Il faut que je me dépêche.

(16) Już wszystko zjadłeś?! 
(16a) Tu as déjà tout mangé?!

Dans (15) już indique qu'il y a un décalage entre l'heure constatée (10h) et l'heure que le locuteur pensait qu'il était (plus tôt que 10h). Il en va de même de (16), dans lequel le point de repère est la fin de la situation de manger. Leurs traductions françaises y sont équivalentes. Les exemples (16) et (16a) s'accompagnent d'une valeur émotive : la surprise (cf. Franckel 1989, Fuchs 1988). Il s'agit ici d'une question rhétorique plutôt que d'une vraie question.

Observons encore avec quels temps verbaux se combinent już et déjà. Le premier énoncé de (15) est averbal. Cet exemple est traduit en français par un énoncé averbal aussi. Pour (16) et sa traduction, la forme verbale a une valeur résultative aussi bien en polonais qu'en français (cf. Apothéloz \& Nowakowska 2010). Il s'agit de l'état résultant de la situation dénotée par la prédication zjeść wszystko (manger tout), ce qui implique que tout est mangé au moment de l'énonciation. Ainsi, le verbe au passé composé indique la résultativité « sémantique », c'est-à-dire qu'on communique l'état résultant qui découle du sens du lexème verbal. Ici, il s'agit non du lexème verbal mais de la prédication manger tout, dans laquelle l'objet direct tout détermine la borne droite. Pour exprimer ce type de résultativité en polonais, on emploie régulièrement non le passé imperfectif mais perfectif (cf. Nowakowska 2008, 2010). En effet, en polonais l'opposition perfectif vs imperfectif sert aussi pour distinguer la résultativité « sémantique », comme dans (16), de la résultativité « pragmatique ». Cette dernière exige un calcul inférentiel et est toujours exprimée par le passé imperfectif en polonais ${ }^{5}$.

\subsection{Już existentiel}

Nous entendons ici par existentiels les emplois dans lesquels już et déjà servent à marquer le caractère effectif d'une situation ${ }^{6}$. Dans ce cas już et déjà ne sont pas absolument indispensables pour produire cette signification. En voici deux exemples :

(17) [w restauracji] Czy panowie już zamawiali ? (exemple de Koschmieder)

(17a) [au restaurant] Est-ce que vous avez déjà commandé?

(18) H. nie chce wracać w ten stan, nie chce ponownie przeżywać choroby. Robi wszystko, żeby wzmocnić nadzieję. Ma pewność, że najgorsze już za nami, że nic ostatecznego nas nie czeka. Drwi z diagnoz i statystyk. (Tuszyńska A., 2007, Ćwiczenia z utraty, 43).

(18a) H. ne veut pas revenir à cet état, il ne veut pas revivre la maladie. Il fait tout pour consolider l'espoir. Il a la certitude que le pire est déjà derrière nous, que rien d'extrême ne nous attend. Il se moque du diagnostic et des statistiques. (Tuszyńska A., 2009, Exercices de la perte, 63; trad. Erhel)

\footnotetext{
${ }^{5}$ Sur la distinction entre résultativité sémantique et résultativité pragmatique voir Apothéloz \& Nowakowska (2010).

${ }^{6}$ Cet emploi se laisse paraphraser aussi par la formule de Franckel (1989) « ce qui est fait n'est plus à faire ».
} 
Dans ces exemples, l'existence ou la non-existence d'une situation concerne des situations présentes ou passées, mais elle peut aussi bien concerner des situations futures.

Quant aux situations passées, les adverbes już et déjà reçoivent une interprétation qui vient de leur combinaison avec le passé imperfectif polonais et le passé composé français. Ces deux formes verbales ont ici une valeur de parfait, que nous appelons « existentiel ». Contrairement à une certaine tradition (Comrie 1976, Karolak 2007), nous distinguons le parfait dit « existentiel » du parfait dit «d'expérience ». La première diffère de la seconde notamment par les propriétés suivantes :

1) Le parfait existentiel désigne une occurrence unique d'une situation, alors que le parfait d'expérience indique que la situation désignée par le verbe a eu lieu une fois au moins.

2) Le parfait d'expérience se distingue du parfait existentiel par sa paraphrase il est arrivé $p$.

3) Déjà / już combinés avec le parfait existentiel sont niés par ne... pas encore / jeszcze nie, alors que ces mêmes adverbes combinés avec le parfait d'expérience ont deux négations possibles ne.. jamais / ne... pas encore / nigdy / jeszcze nie (Nowakowska 2008 : 167-169).

Nous retrouvons ces propriétés dans l'exemple (17) et sa traduction française, qui illustrent le parfait existentiel, et dans un exemple français cité plus haut que nous reprenons ici pour illustrer le parfait d'expérience. Observons surtout leur troisième propriété :

(17b) - Czy panowie już zamawiali ?

- Nie, jeszcze nie (*nie, nigdy).

(17c) - Est-ce que vous avez déjà commandé?

- Non, pas encore. (*non, jamais).

(10a) - Tu as déjà mangé des blinis?

- Non jamais / Non, pas encore.

(10b) - Czy już jadłeś bliny ?

- Nie, nigdy. / Nie, jeszcze nie.

L'imperfectif polonais à valeur de parfait existentiel combiné avec już et le passé composé ayant cette même valeur combiné avec déjà pourraient être décrits au moyen de la notion de scénario. Cela signifie que już ou déjà accompagnent ces formes verbales lorsqu'elles dénotent une situation qui s'inscrit dans une séquence de situations (scénario) (Nowakowska 2008). Ces situations suivent habituellement un certain ordre comme celui des actions effectuées par les participants du scénario « au restaurant» (choisir les plats, commander, manger, payer). La question (17) et (17a) est posée par le serveur pour savoir si l'étape de la commande est réalisée ou non. La réponse plausible dans cette situation est par exemple «C'est fait » ou «Pas encore ». 
En réalité, ce qui caractérise les situations concernées par już et déjà existentiels, ce qu'elles sont attendues. Celles qui interviennent dans un scénario ne sont qu'un cas particulier des situations attendues.

Comme on l'observe dans (17), la valeur de parfait existentiel se réalise en polonais par le passé imperfectif et non par le passé perfectif. Le caractère étrange ou surprenant de ce choix a été remarqué par Koschmieder (1934 : 21), qui se demandait pourquoi une situation entièrement réalisée est exprimée ici par l'imperfectif.

Avant d'analyser (18), remarquons que dans la subordonnée qui comprend $j u \dot{z}$, il y a ellipse du verbe być (être). Cela ne change pas vraiment notre analyse : l'ellipse est faite parce qu'on a affaire à la situation liée au nunc. Nous pouvons donc communiquer le même par un énoncé verbal : ... najgorsze jest już za nami (... le pire est déjà derrière nous). Ainsi, nous allons traiter cet emploi de już comme s'il se combinait avec une forme verbale au présent.

Après ces constats préliminaires, passons à l'analyse de (18) et (18a). Ces exemples diffèrent des deux précédents par le temps du verbe. Cependant, il s'agit ici du même emploi de już et de déjà que précédemment : les deux adverbes marquent l'existence de la situation dénotée par la forme verbale. Dans les exemples (18) et (18a) il est question d'une maladie. Le constat selon lequel le pire est déjà derrière nous renvoie à un micro-scénario dans lequel la maladie est généralement suivie d'une amélioration.

\subsection{Już itératif-continuatif}

Cet emploi de już et de déjà apparaît lorsque le verbe avec lequel ils se combinent désigne une situation, tout en indiquant que cette situation s'est déjà produite (itération) ou était déjà le cas (continuation) à un moment antérieur. Autrement dit, il s'agit de la seconde occurrence d'une situation ou de l'état antérieur qui persiste. On peut donc gloser cet emploi par : « Ce n'est pas nouveau ». Les deux exemples suivants illustrent ces deux variantes :

(19) Nie jestem sportowcem, już ci mówiłem. I nie zgadzam się pływać w słodkiej wodzie.

(19a) Je ne suis pas un sportif, je te l'ai déjà dit. Et je refuse de nager en eau douce. (Pontalis J.-B., En marge des jours, 2002)

(20) Wytłumaczyła mi, jak się umyć, a potem jak się kochać... [alinéa] Ja już to wiedziałem, ale dałem jej mówić, żeby się lepiej czuła, zresztą podobał mi się jej głos, trochę nadąsany, trochę zasmucony. (Schmitt E.-E., 2010, Pan Ibrahim $i$ kwiaty Koranu, p. 8 ; trad. Grzegorzewska)

(20a) Elle m'a expliqué comment on se lavait, puis comment on devait faire l'amour... [alinéa] Évidemment, je savais déjà mais je la laissais dire, pour qu'elle se sente plus à l'aise, et puis j'aimais bien sa voix, un peu boudeuse, un peu chagrinée. (Schmitt E.-E., 2001, Monsieur Ibrahim et les Fleurs du Coran, p. 11) 
L'énoncé qui comprend już et déjà des exemples (19)-(19a) signifie que l'action de dire se répète au moment de l'énonciation et celui des exemples (20)-(20a) signifie que l'état de savoir continue au point de référence donné par le texte. Remarquons que si l'on effaçait ces adverbes dans ces exemples respectifs le sens de répétition et celui de continuation disparaîtrait.

\subsection{Już de parfait d'expérience}

Comme nous l'avons vu plus haut (cf. § 2.2.), cet emploi découle de la valeur de la forme verbale. Il s'agit du parfait d'expérience qui a été étudié surtout pour l'un des emplois du Present Perfect anglais (Leech 1971, Comrie 1976, McCawley 1971), mais aussi pour le bulgare (cf. Guentchéva 1990, Karolak 1997). Certains linguistes ont remarqué que cette même valeur peut caractériser le passe composé français (cf. Vet 1992, Desclés \& Guentchéva, Karolak 2007, Apothéloz \& Nowakowska 2010, Apothéloz 2010b) et le passé surcomposé dit « régional » français (Apothéloz 2009, 2010a, à par.). Le polonais emploie dans ce cas le passé imperfectif (Karolak 2007, 2008, Apothéloz \& Nowakowska 2010).

Cette valeur se caractérise grosso modo par l'indétermination de la localisation temporelle, la neutralisation du nombre d'occurrences de la situation dénotée par le verbe, par la présence d'une variable prédicative de type $I l$ est arrivé $p$. Cette variable prédicative implique un intervalle temporel de validation.

Les formes verbales ayant la valeur de parfait d'expérience peuvent s'accompagner de différentes marques aspectuo-temporelles comme kiedykolwiek (jamais), nigdy (jamais), kilka razy (plusieurs fois), etc. Ce qui nous intéresse, c'est qu'ils s'accompagnent aussi de l'adverbe już / déjà, comme nous l'observons ci-dessous :

(21) Ona już była (kilka razy) za granica, więc poradzi sobie.

(21a) Elle est déjà allée (plusieurs fois) à l'étranger, donc elle se débrouillera.

(22) Tak, już jeździłam na nartach.

(22a) Oui, j'ai déjà fait du ski.

(23) - [.. ] Czy Pani już otwierała podobną butelkę?

- Nie, nigdy, powiedziałam, [...]

(23a) - [...] Vous avez déjà débouché une bouteille pareille?

- Non, jamais, ai-je dit, [...]. (Brissac, Frantexte)

Contrairement aux précédents, qui sont niés seulement par jeszcze nie /ne... pas encore, cet emploi de juz / déjà est nié par nigdy / ne.. jamais ou par jeszcze nie / ne... pas encore. On notera à propos de l'exemple (21) que quand la valeur d'expérience concerne l'idée de déplacement, le polonais, comme l'anglais, utilise le verbe być (être) et non un verbe de mouvement.

Pour terminer cette section, il est intéressant d'observer que le rapport entre la présence de juz / déjà et les quatre valeurs décrites ci-dessus n'est pas le même selon la valeur considérée. Alors que l'adverbe est indispensable pour produire la valeur 
de survenance précoce et celle d'itération-continuation, les valeurs existentielle et de parfait d'expérience peuvent fort bien être exprimées sans ces adverbes. Dans ce cas, już et déjà fonctionnent comme de sortes de spécificateurs; ils servent alors à désambiguïser l'énoncé et à sélectionner la lecture respectivement existentielle ou de parfait d'expérience. Ainsi, sans już / déjà et moyennant un contexte adéquat, (17) et (18) peuvent parfaitement être interprétés comme existentiels et (21), (22) et (23), comme expérientielle.

\section{Non-correspondance entre $j u \dot{z}$ et déjà}

Après la présentation des cas où już polonais correspond à déjà français, passons maintenant à l'examen de cas dans lesquels już ne peut pas être traduit par dejà. Vu la complexité de la question, nous nous limiterons à quelques emplois de $j u \dot{z}$.

\subsection{Już en contexte négatif}

Dans deux cas au moins już ne peut pas être traduit par déjà : dans les séquences

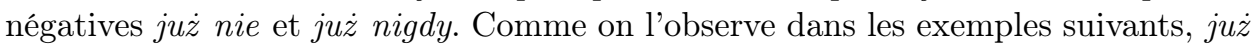
nie est traduit par ne... plus et już nigdy par plus jamais :

(24) Już nie mamy mąki. Trzeba iść do sklepu.

(24a) Nous n'avons plus de farine. Il faut aller au magasin.

(25) Nie będe prowadzit wozu.

Ty - pasjonat motoryzacji [...] - teraz nonszalancko mówisz:

Trudno. Już nigdy. Sa gorsze zmartwienia. (Tuszyńska A., 2007, Ćwiczenia $z$ utraty, 15)

(25a) Je ne conduirais plus de voiture.

Toi, passionné de moteurs [...], maintenant tu dis nonchalamment : Rien

à faire. Plus jamais. Il y a des soucis bien pires. (Tuszyńska A., 2009, Exercices de la perte, 23 ; trad. Erhel)

Concernant l'exemple (24) on pourrait certes introduire dans sa traduction un déjà (Nous n'avons déjà plus de farine), mais cette formulation diffère sémantiquement de (24a) car déjà introduit la valeur de survenance précoce, absente de l'exemple polonais. Il est intéressant d'observer que le polonais ne peut pas marquer la survenance précoce en contexte négatif.

\subsection{Już en emploi prospectif}

L'un des emplois de już non traductibles par déjà combine l'immédiateté avec l'indication du moment de l'énonciation. L'immédiateté est limitée à un intervalle qui est borné à gauche par le nunc et ouvert à droite. On peut donc dire que ce już accentue le caractère imminent d'une situation postérieure au nunc. C'est pourquoi il sera désormais qualifié de «prospectif ». Déjà est inapte à traduire cet emploi 
de $j u \dot{z}$. Des traductions possibles mais approximatives sont bientôt, tout de suite, immédiatement ou maintenant.

Dans cet emploi juz peut se combiner avec un verbe au futur et / ou avec des localisateurs temporels comme zaraz (dans un instant). L'exemple suivant illustre ce qui vient d'être dit. La combinaison juz zaraz y a été traduite par l'adverbe tout de suite.

(26) Neurochirurgia, Toronto, ciemnoskóra pielęgniarka o egzotycznym imieniu Princess, pragnienie, pić, nie można, już-już zaraz, chirurg kończy poprzedniego, trzecia, piąta godzina czekania. (Tuszyńska A., 2007, Ćwiczenia z utraty, 60)

(26a) Neurochirurgie, Toronto, infirmière noire au prénom exotique de Princess, soif, boire, impossible, voilà, voilà, tout de suite, le chirurgien finit avec le précédent, troisième, cinquième heure d'attente. (Tuszyńska A., 2009, Exercices de la perte, 85 ; trad. Erhel)

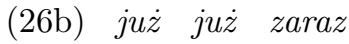

(26c) ? ? dans un instant ${ }^{7}$

La suppression de zaraz ne modifierait en rien le sens de l'énoncé, ce qui paraît confirmé ici que już cumule la référence au nunc et l'indication d'immédiateté. Le fait que $j u \dot{z}$ soit ici redoublé ne change rien à notre raisonnement, ce redoublement n'étant qu'une simple marque de renforcement.

Examinons maintenant cet emploi de już combiné avec un verbe au futur et un verbe modal :

(27) Krzewy już, już pokryją się liśćmi. ( $\left.\mathrm{SJP}^{8}\right)$

(27a) Les arbrisseaux vont bientôt se couvrir de feuilles.

(27c) arbrisseaux? ? se couvrir(FUTUR) de feuilles

(28) Chirurg precyzyjnie dobiera słowa ostateczne. Mają nam pomóc w uporządkowaniu świata. Doczesność może już wkrótce przestać nas dotyczyć. (Tuszyńska A., 2007, Ćwiczenia z utraty, 6)

(28a) Le chirurgien choisit avec précision des mots définitifs. Ils doivent nous aider à mettre le monde en ordre. Le monde „terrestre” peut bientôt cesser de nous concerner. (Tuszyńska A., 2009, Exercices de la perte, 10 ; trad. Erhel)

$\begin{array}{lllllll}\text { (28c) doczesność } & \text { może już } & \text { wkrótce } & \text { przestać } & \text { nas dotyczyć } \\ \text { (28d) monde „terrestre” } & \text { peut ? } & \text { bientôt } & \text { cesser } & \text { nous concerner }\end{array}$

\footnotetext{
${ }^{7}$ Dans les gloses métalinguistiques le point d'interrogation signale seulement la nontraductibilité de już par déjà.

8 Stownik Języka Polskiego PWN.
} 
Dans (27) już est employé seul, alors que dans (28) il se combine avec l'adverbe wkrótce (bientôt). Le sens de (27) ne changerait pas si l'on y ajoutait wkrótce (bientôt) et le sens de (28) ne changerait pas non plus si on effaçait wkrótce (bientôt). Dans ce contexte już et już wkrótce sont équivalents ${ }^{9}$, tout comme étaient équiva-

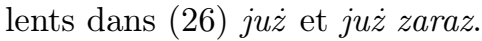

Observons encore deux exemples qui comportent juz prospectif :

(29) Stefan - krzyknęła słabym głosem matka - idziesz już?!

- Tak - powiedział - idę już, kochanie. (Hłasko, Ósmy dzień tygodnia, p. 17

(29a) Stefan - s'écria la mère d'une voix faible - tu t'en vas?

- Oui - dit-il - je m'en vais tout de suite / j'y vais j'y vais/, chérie.

(30) - Dlaczego Pani nie pije?

- Już piję (Hłasko, Ósmy dzień tygodnia, p. 85)

(30a) - Pourquoi vous ne buvez pas?

- Je bois, je bois / Je bois tout de suite.

(29) présente un dialogue : la question et la réponse concernent le fait que l'un des deux interlocuteurs devait sortir. Ce dialogue comporte deux emplois de $j u \dot{z}$. Le premier combine les valeurs déictique et existentielle et sera examiné plus bas sous l'appellation de $j u \dot{z}$ 《rétrospectif ». Le second est un emploi prospectif. En effet, le locuteur de la réponse rassure son interlocuteur en lui disant qu'il partira immédiatement. L'adverbe français déjà ne convient pas ici : il produirait le sens de survenance précoce, ce qui est complètement étranger à l'énoncé polonais. Cela montre bien que la survenance précoce et l'immédiateté déictique sont deux notions différentes. Cet emploi de juz peut être traduit par des adverbes comme tout de suite ou immédiatement, voire par le redoublement de l'énoncé, comme dans (29a) et (30a). Dans ces exemples le locuteur accomplit un acte de type promissif au sens de Searle (1982). Notons que juz prospectif ne peut être enlevé sans disparition de l'idée d'immédiateté.

La traduction suivante, extraite d'un manuel de polonais, est donc sinon incorrecte du moins approximative :

(31) - Pamiętasz o której włożyłyśmy ciasto do pieca?

- W pół do drugiej.

- To trzeba je już wyjąć.

(31a) - Tu te souviens à quelle heure nous avons mis le gâteau dans le four?

- A une heure et demie.

- Alors, il faut déjà le sortir.

\footnotetext{
${ }^{9}$ Bientôt est neutre du point de vue de l'emploi déictique ou anaphorique (cf. Tahara 2006).
} 
Déjà dans cette traduction ne peut être interprété que comme signifiant la survenance précoce, or ce n'est du tout de cela qu'il s'agit dans le texte polonais.

\subsection{Injonction renforcée}

Le juz prospectif apparaît aussi dans des énoncés qui réalisent des actes directifs. Il sert donc à renforcer le caractère immédiat de la réalisation d'une situation. Considérons l'exemple suivant :

(32) Wyłącz to już !

(32a) Débranche ça tout de suite!

Comme précédemment, la traduction la plus proche est tout de suite ou un adverbe équivalent. Cependant, s'agissant d'un acte directif, on pourrait penser ici à une traduction de juz par enfin exclamatif. Mais, trois raisons au moins conduisent à rejeter cette traduction. D'une part, enfin exprime l'impatience, ce que ne fait pas już, d'autre part enfin exclamatif a un fonctionnement méta-énonciatif, ce qui n'est pas le cas de już, en troisième lieu, enfin laisse entendre que la même injonction a été produite antérieurement, élément totalement absent de $j u \dot{z}$.

Le juz prospectif intervient aussi dans d'autres formes d'énonces directifs, par exemple, dans les indépendantes injonctives, comme dans (33), ou des énoncés averbaux, comme dans (34) :

(33) [...] niechby już mnie przyjęli, [...] (M. V. Llosa, 2008, Rozmowa w Katedrze, p. 73).

(33a) [...] qu'ils m'acceptent immédiatement, [...]

(34) - Dość już tych kpin - Espina uderzył ręką w stół. (M. V. Llosa, 2008, Rozmowa w Katedrze, p. 67)

(34b) - Ça suffit maintenant, ces moqueries - Espina frappa de la main sur la table.

(34c) dość już tych kpin

(34d) assez? ces moqueries (GÉNITIF)

Dans les énoncés directifs $j u \dot{z}$ prospectif ne peut pas être traduit par déjà. Les traductions possibles sont maintenant, tout de suite ou immédiatement.

\subsection{Już en emploi rétrospectif}

Cet emploi de juź ressemble à l'emploi « existentiel » que déjà et juz partagent, que nous avons présenté supra (cf. § 2.2.) : il marque l'existence ou la non-existence d'une situation attendue. Mais, l'emploi rétrospectif de juz diffère de l'emploi existentiel par son lien avec le moment de l'énonciation. Il se combine donc avec un verbe désignant une situation qui a lieu au moment de l'énonciation ou juste avant. Nous appelons ce $j u \dot{z}$ 《rétrospectif » parce qu'il faut une vision rétrospective pour 
constater l'existence ou la non-existence d'une situation. L'exemple suivant, où il est question d'une marche vers un lac, montre que cet emploi de $j u \dot{z}$ ne peut pas être traduit par déjà :

(35) (Niżej i znowu niżej, dwanaście kolejnych kroków. [...] Już widać wodę. Cały czas było ją widać, ale jest bliżej. Już można się powołać na jej autorytet. (Tuszyńska A., 2007, Ćwiczenia z utraty, 226).

(35a) Plus bas, et encore plus bas, douze nouveaux pas. [...] Maintenant on voit l'eau. On la voyait tout le temps mais elle est plus proche. On peut maintenant s'en remettre à son autorité. (Tuszyńska A., 2009, Exercices de la perte, 301 ; trad. Erhel)

La traduction de juz par maintenant rend d'une certaine façon le sens de $j u \dot{z}$. Comme już, maintenant invite à opposer ce qui se passe au nunc à ce qui se passait à l'époque antérieure. Mais l'adverbe już laisse également entendre qu'il était attendu que les protagonistes arrivent auprès d'un lac, ce que ne fait pas maintenant. Cette dernière idée pourrait être restituée par un déjà de « consécution narrative »au sens de Weinrich (1973) (Déjà on voit l'eau). Mais cet emploi de déjà véhicule également le sens de survenance précoce, sens absent dans l'énoncé polonais.

L'absence de correspondance entre już rétrospectif et déjà provoque des fautes de traductions. En voilà une : il s'agit d'un dépliant pour touristes qui annonce l'ouverture du Musée de Sukiennice de Cracovie :

(36) Muzeum w Sukiennicach już otwarte! (dépliant, novembre 2010)

(36a) Le musée de Sukiennice est déjà ouvert! (dépliant novembre 2010, version française)

(36b) Le musée de Sukiennice vient juste d'ouvrir!

(36c) Le musée de Sukiennice est désormais ouvert!

$\begin{array}{llll}\text { (36d) Muzeum } & w & \text { Sukiennicach już } & \text { otwarte } \\ \text { (36e) Musée } & \text { PRÉP. Sukiennice désormais ouvert }\end{array}$

La traduction (36a) ne convient pas dans cette situation parce qu'on devrait comprendre le message polonais comme «plus tôt que prévu», ce qui n'est pas le cas. D'ailleurs, en réalité on a mis beaucoup de temps pour restaurer ce musée. L'idée de survenance précoce aurait pu donc avoir un sens ironique. Or, dans (36) on communique seulement l'existence d'une situation récente ou sa réalisation récente. Ainsi, ce message se laisse traduire en français par (36b), dans lequel on souligne le caractère récent de l'ouverture du musée par un verbe au passé récent et par l'adverbe juste. Ce dernier marque la coïncidence avec le moment de l'énonciation. Mais, cette traduction « trahit » néanmoins la prédication polonaise qui est à la voix passive. Dans ce sens la traduction par (36c) est meilleure : elle comporte aussi la voix passive. L'adverbe désormais convient bien ici parce qu'il marque un point 
de référence, qui est ici le nunc, et l'ouverture de l'intervalle à droite. Néanmoins, ce que ne marque pas désormais, c'est l'idée d'attente.

Considérons encore un exemple de juz rétrospectif. Ici aussi, l'adverbe już peut être traduit par désormais ou une de ses variantes comme d'or et déjà, dès maintenant :

(37) Słownik Oxforda już tylko w Internecie [titre]. (Metro, 9.09.2010)

(37a) Le dictionnaire Oxford désormais /d'or et déjà / dès maintenant seulement sur Internet.

$(37 \mathrm{c})$ dictionnaire Oxford ? seulement sur Internet

Cet exemple invite à deux commentaires. Premièrement, le $j u \dot{z}$ polonais s'identifie discursivement à désormais, d'or et déjà ou dès maintenant. Comme nous le savons, ces adverbiaux marquent à la fois le lien avec le nunc et l'ouverture à droite de l'intervalle considéré. Deuxièmement, la traduction par déjà est doublement exclue : par son sens de survenance précoce et l'impossibilité de combiner déjà avec seulement. Cette dernière découle probablement du fait que seulement a aussi un sens temporel, sens qui ne s'accorde pas avec celui de déjà.

Notons que l'exemple (37) peut être aussi interprété comme już de survenance précoce. Cette interprétation est plausible dans le contexte suivant : le locuteur exprime sa surprise quant à la numérisation des livres. Suivant ce raisonnement, on comprend que l'apparition de ce dictionnaire sur Internet ne représente pas un regret vis-à-vis du livre traditionnel mais la satisfaction de la création des livres purement numériques. Dans ce cas, on traduirait cet exemple par déjà :

(37d) Le dictionnaire Oxford n'est déjà plus que sur Internet.

$\mathrm{Vu}$ l'analyse des traductions de już rétrospectif, on constate qu'il correspond à désormais ou une de ses variantes comme d'or et déjà, dès maintenant, mais ces adverbes sont dépourvus de l'idée d'attente.

\subsection{Juz polyphonique}

Il existe un autre emploi de $j u \dot{z}$ qui est difficile à décrire. Il se combine avec l'adverbe zawsze (toujours) ou des expressions de durée comme do (jusqu'à) + NOM DE LOCALISATION TEMPORELlE. Bien que ce już prenne dans sa portée une expression temporelle, il a un caractère non temporel. Nous l'appelons « polyphonique » parce qu'il exige de concevoir deux points de vue dans un seul énoncé. Cela signifie qu'il introduit une sorte de confrontation avec un autre point de vue et sert ainsi à rejeter un point de vue différent de celui qui est exprimé par l'énoncé. Juz dans ce contexte marque, nous semble-t-il, une sorte d'insistance sur l'assertion de la durée de la situation. Le już polyphonique ne peut pas être traduit par déjà :

(38) [...] zmuszony był oddać żonę do zakładu, gdzie pozostała już do śmierci. (Fry - Wystawa Grupy Bloomsbury, 2010). 
(38a) ([.. ] il fut contraint à laisser sa femme dans une institution, où elle resta jusqu'à sa mort.

$\begin{array}{lllll}\text { gdzie pozostała } & \text { juz } & \text { do } & \text { śmierci. } \\ \text { où } & \text { resta (FÉMININ) } & ? & \text { jusqu'à mort }\end{array}$

Dans cet exemple, już introduit l'idée de l'élimination du point de vue selon lequel on peut douter si la personne dont on parle reste dans une institution effectivement jusqu'à sa mort. Si l'on efface już, on obtient un énoncé acceptable et naturel, mais cet énoncé sera dépourvu de son caractère polyphonique. En français on obtiendrait un effet polyphonique similaire avec des adverbes comme vraiment / bel et bien / effectivement.

Il en va de même des exemples suivants, qui comportent la combinaison de już avec zawsze (toujours) :

(39) W czwartek wieczór wróciliśmy z Rzymu; z tego innego, prywatnego życia, które od teraz zamierzam wieść już zawsze. (W. Woolf, Chwile wolności, Dziennik 1915-41, p. 321).

(39a) Jeudi soir nous sommes rentrés de Rome; de cette vie différente, privée, que désormais j'envisage de mener pour toujours.

(39b) które od teraz zamierzam wieść już zawsze.

(39c) que depuis maintenant voudrais (je) mener ? toujours.

(40) Wysiadając, osuwasz nogi na ziemię. Tak jest już teraz zawsze, kiedy długo nie zmieniasz pozycji. Ignorujesz to zupełnie. Jak bezwład nóg, jak zawroty głowy. (Tuszyńska A., 2007, Ćwiczenia z utraty, 225).

(40a) En descendant tu t'affaisses sur le sol. Il en est toujours ainsi maintenant lorsque tu restes longtemps sans changer de position. Tu l'ignores complètement. Comme la paralysie des jambes, comme les vertiges. (Tuszyńska A., 2009, Exercices de la perte, 299; trad. Erhel)

(40b) Tak jest jü teraz zawsze

(40c) ainsi (c')est ? maintenant toujours.

Ici, już implique que le point de vue contraire est rejeté, point de vue selon lequel la situation durera moins longtemps que toujours. Observons que dans ces exemples już n'est pas du tout traduit. Ainsi, dans la traduction de (39), l'adverbe désormais traduit l'adverbe polonais od teraz et non już et dans la traduction de (40) l'adverbe maintenant traduit teraz et non juz. Notons enfin que dans ces deux traductions il est possible de traduire już par l'un des adverbes français que nous venons de citer, vraiment / bel et bien / effectivement. 


\section{Conclusion}

Nous avons effectué une analyse qui a visé à comparer l'adverbe już polonais avec l'adverbe déjà français. Dans la première section du présent article, nous avons traité de la correspondance entre ces deux adverbes. Les adverbes już et déjà partagent les quatre emplois temporels suivants : emploi de survenance précoce, existentiel, itératif-continuatif et de parfait d'expérience. Dans la seconde section, nous avons passé en revue les cas dans lesquels już ne peut être traduit par déjà.

Premièrement, nous avons observé que les séquences négatives juz nie et juz nigdy ne se traduisent pas par déjà mais par ne... plus et ne jamais. De plus, la séquence polonaise już nie n'est pas apte à rendre la valeur de survenance précoce, valeur qui est exprimée en français par déjà plus.

Deuxièmement, nous avons montré que już a conventionnalisé un sens déictique, ce qui n'est pas le cas de déjà. Ce sens est présent dans deux emplois de już: prospectif et rétrospectif.

Quand już est employé de façon prospective d'autres adverbes que déjà peuvent plus au moins bien en traduire les effets sémantiques : tout de suite, immédiatement, bientôt et maintenant. Un autre moyen de traduction possible est le redoublement de l'énoncé ( $j^{\prime} y$ vais, j'y vais). Dans les énoncés directifs, qui comportent aussi un $j u \dot{z}$ prospectif, nous avons montré qu'il n'est pas possible de le traduire par enfin exclamatif. Ce dernier exprime l'impatience du locuteur, qui accompagne sans doute les énoncés directifs de ce type mais n'exprime pas vraiment le sens produit par $j u \dot{z}$. Dans ce cas już n'a pas le fonctionnement méta-énonciatif de enfin exclamatif. Bref, już prospectif dans tous ces contextes n'est jamais traduit par déjà.

Dans son emploi rétrospectif, $j u \dot{z}$ peut être traduit assez fidèlement par des adverbes comme maintenant, désormais (d'or et déjà, dès maintenant) ou bien par juste combiné avec un verbe au passé récent. Néanmoins, ces adverbes, contrairement à $j u \dot{z}$, ne fonctionnent pas dans l'univers de l'attendu.

Troisièmement, nous avons montré qu'il existe un emploi polyphonique de $j u \dot{z}$, non traductible par déjà, mais trouvant des équivalents dans vraiment, bel et bien et effectivement. Ce dernier emploi de już a un fonctionnement argumentatif dans le texte. Il n'a donc pas de caractère temporel bien qu'il se combine avec des expressions de durée.

Le présent article constitue une petite contribution à la description de już et de déjà. Il ne comporte que des résultats fragmentaires d'une recherche actuellement en cours.

\section{Références}

Apothéloz, Denis (2009). La quasi-synonymie du passé composé et du passé surcomposé dit «régional». Pratiques, no 141/142, 98-120.

Apothéloz, Denis (2010a). Le passé surcomposé et la valeur de parfait existentiel. Journal of French Language Studies, vol. 20, no 2, 105-126.

Apothéloz, Denis (2010b). De l'usage argumentatif du parfait d'expérience. In Górnikiewicz, Joanna, Grzmil-Tylutki, Halina \& Piechnik, Iwona (éds), En quête de sens. $W$ poszukiwaniu znaczeń. Etudes dédiées à Marcela Światkowska. Studia dedykowane Marceli Świątkowskiej. Wydawnictwo UJ, Kraków, 29-38. 
Apothéloz, Denis (à paraître). La concurrence du passé composé et du passé surcomposé dans l'expression de la valeur de parfait d'expérience. Actes de la Conférence 2009 de l'Association for French Language Studies, Neuchâtel, 3-5 sept. 2009.

Apothéloz, Denis \& Nowakowska, Małgorzata (2010). La résultativité et la valeur de parfait en français et en polonais, Cahiers Chronos, 21, 1-23.

Apothéloz, Denis \& Nowakowska, Małgorzata (à par. a). Déjà en emploi justificatif. A paraître dans un volume d'hommage.

Apothéloz, Denis \& Nowakowska, Małgorzata (à par. b). Les emplois temporels de déjà. In Actes du colloque Chronos 10, Birmingham, 2011.

Bańkowski, Andrzej (1971). Jeszcze i już, Język Polski, LI, 21-30.

Bańkowski, Andrzej (1976). Opozycja semantyczna partykuł dopiero i już, Prace filologiczne, XXVI, 13-38.

Bańkowski, Andrzej (1977). Opozycja semantyczna partykuł jeszcze i już, Studia językoznawcze. Streszczenia prac doktorskich, II, (Prace językoznawcze, 88), Wrocław, $7-46$.

Buchi, Eva (2007). Approche diachronique de la (poly)pragmaticalisation de français déjà ("Quand le grammème est-il devenu pragmatème, déjà?"), In Trotter, David (éd.), Actes du XXIV ${ }^{e}$ Congrès international de Linguistique et de Philologie Romanes (Aberystwyth), Niemeryer, Tübingen, $3: 251-264$.

Comrie, Bernard (1976). Aspect. An introduction to the study of verbal aspect and related problems, Cambridge University Press, London-New York - Melbourne.

Desclés, Jean-Pierre \& Guentchéva, Zlatka (2003). Comment déterminer les significations du passé composé par une exploration textuelle?, Langue Française, 138, 48-60.

Franckel, Jean-Jacques (1989). Étude de quelques marqueurs aspectuels du français, Droz, Genève-Paris.

Fuchs, Catherine (1988). Encore, déjà, toujours : de l'aspect à la modalité, In Tersis, Nicole \& Kihm, Alain (éds), Temps et aspects, Actes du colloque CNRS, Paris, 24-25 octobre 1985, Peeters \& SELAF, Louvain - Paris, 136-148.

Grochowski, Maciej (1986). Polskie partykuły. Składnia, semantyka i leksykografia, Ossolineum, Wrocław.

Grzegorczykowa, Renata (1975). Funkcje semantyczne i sktadniowe polskich przysłówków, Ossolineum, Wrocław.

Guentchéva, Zlatka (1990). Temps et aspect : l'exemple du bulgare contemporain, Editions du CNRS, Paris.

Hoepelman, Franz J. \& Rohrer, Christian (1980). Déjà, encore et les temps du passé en français, In David, Jean \& Martin, Robert (éds), La notion d'aspect, (coll. « Recherches linguistiques V »), Centre d'Analyse syntaxique de l'Université de Metz, Metz, 119-143.

Karolak, Stanisław (1997). Le temps et le modèle de H. Reichenbach, Etudes Cognitives / Studia kognitywne, 2, 95-125.

Karolak, Stanisław (2007). Wyrażenia predykatywne orzeczeniowe, In Składnia francuska o podstawach semantycznych, tom 1, Collegium Columbinum, Kraków, 33-54.

Karolak, Stanisław (2008). Remarques sur l'équivalence du passé imperfectif polonais et des temps passés en français, Verbum, 30, 2-3, 125-146.

Koschmieder, Erwin (1934). Nauka o aspektach czasownika polskiego w zarysie. Próba syntezy. Rozprawy i materiały Wydziału i Towarzystwa Przyjaciół Nauk w Wilnie, tom V, zeszyt 2, Towarzystwo Przyjaciół Nauk w Wilnie, Wilno.

Leech, Geoffrey N. (1971). Meaning and the English Verb, Longman, London. 
Martin, Robert (1980). Déjà et encore : de la présupposition à l'aspect, In David Jean et Martin Robert (éds), La notion d'aspect, (coll. «Recherches linguistiques V»), Centre d'Analyse syntaxique de l'Université de Metz, Metz, 167-179.

McCawley, James D. (1971). Tense and time reference in English, in : Fillmore, Charles J. \& Langendoen, Terence D. (Eds), Studies in linguistic semantics, Holt, Rinehart and Winston, New York, 96-113.

Mosegaard Hansen, Maj-Britt (2000). La polysémie de l'adverbe déjà, dans : (éds), Andersen, Leth H. \& Hansen, Anita B., Le français parlé. Actes du colloque international. Université de Copenhague du 29 au 30 octobre 1998 (Etudes romanes 47), $157-178$.

Mosegaard Hansen, Maj-Britt (2002a). From Aspectuality to Discourse Marking : the Case of French déjà and encore, Belgian Journal of Linguistics, 16, 23-51.

Mosegaard Hansen, Maj-Britt (2002b). La polysémie de l'adverbe encore, Travaux de linguistique, 44, 143-166.

Mosegaard Hansen, Maj-Britt (2008). Particles at the semantics/pragmatics interface : synchronic and diachronic issues : a study with special reference to the French phasal adverbs, Elsevier, Amsterdam.

Muller, Claude (1975). Remarques syntactico-sémantiques sur certains adverbes de temps, Le Français moderne, 43, 12-38.

Nowakowska, Małgorzata (2008). L'emploi « paradoxal » de l'imperfectif passé polonais et ses correspondants en français, Verbum, 30.2-3, 147-180.

Nowakowska, Małgorzata (2010). Czasowniki « dwukierunkowe » w użyciu perfektowym, In Górnikiewicz, Joanna Grzmil-Tylutki, Halina \& Piechnik, Iwona (éds), En quête de sens. W poszukiwaniu znaczeń. Etudes dédiées à Marcela Świattkowska. Studia dedykowane Marceli Światkowskiej, Wydawnictwo UJ, Kraków, 29-36.

Nøjgaard, Morten (1992-95). Les adverbes français. Essai de description fonctionnelle, 3 volumes, Munksgaard, Copenhagen.

Paillard, Denis (1992). Déjà et la construction de l'énoncé, L'information grammaticale, $55,33-37$.

Pasicki, Adam (1976). „Już”, ,jeszcze” and their English Equivalents, Papers and Studies in Contrastive Linguistics, V, 103-110.

Searle, John R. (1982). Sens et expression. Etudes de théorie des actes de langages, Minuit, Paris.

Tahara, Izumi (2006). Adverbes temporels et point de vue : le cas de déjà et bientôt, TRANEL (Travaux neuchâtelois de linguistique), 45, 99-113.

Vet, Co (1992). Le passé composé : contexte d'emploi et interprétation, Cahiers de praxématique «Le passé composé », 19, 37-59.

Weinrich, Harald (1973). Temps, Seuil, Paris.

Wierzbicka, Anna (1969). Problemy ekspresji. Ich miejsce w teorii semantycznej, In Dociekania semantyczne, Ossolineum, Wrocław. 